July, 1993

IFT-P.038/93

hep-th/9307064

\title{
On Non-linear W-Infinity Symmetry of Generalized Liouville and Conformal Toda Models
}

\author{
H. Aratyn] \\ Department of Physics \\ University of Illinois at Chicago \\ 845 W. Taylor St. \\ Chicago, Illinois 60607-7059 \\ L.A. Ferreiraf, J.F. Gomes ${ }^{2}$, and A.H. Zimerman ${ }^{2}$ \\ Instituto de Física Teórica-UNESP \\ Rua Pamplona 145 \\ 01405-900 São Paulo, Brazil
}

\begin{abstract}
Invariance under non-linear $\hat{W}_{\infty}$ algebra is shown for the two-boson Liouville type of model and its algebraic generalizations, the extended conformal Toda models. The realization of the corresponding generators in terms of two boson currents within KP hierarchy is presented.
\end{abstract}

\footnotetext{
${ }^{1}$ Work supported in part by U.S. Department of Energy, contract DE-FG02-84ER40173 and by NSF, grant no. INT-9015799

${ }^{2}$ Work supported in part by $\mathrm{CNPq}$
} 


\section{Introduction}

Recently there has been an increasing interest in applications of W-infinity algebras in various areas of theoretical physics, like strings, matrix models, Hall effect etc. The W-infinity symmetry appears in two related forms, the linear $W_{1+\infty}$ algebra, which arises as a deformation of the area preserving symmetry and its non-linear deformation, so-called $\hat{W}_{\infty}$ algebra. These algebraic structures find their natural realization in the setting of the first and second

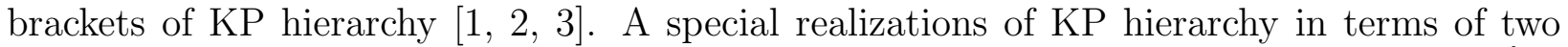
bosons have been proposed in literature 4 , 5. In this paper we work with realization of $\hat{W}_{\infty}$ within this context, using two Bose KP currents as building blocks of the $\hat{W}_{\infty}$ generators.

Despite of the increasing importance of $\mathbf{W}$-infinity algebras the number of non-trivial (interacting) field theoretical models displaying this type of symmetry remains very limited. The aim of this paper is to provide non-trivial invariant actions under the non-linear $\hat{W}_{\infty}$ symmetry.

One example of previously known model exhibiting W-infinity invariance is provided by the conformal affine Toda (CAT) model [5]. The CAT model arises from the affine Toda model by introducing two extra (ghost) fields, which makes it conformally invariant [6, 7]. These extra ghost fields bear responsibility for enlargement of symmetry.

In a similar manner one can extend the conformal symmetry of the Liouville and Toda conformal actions by introducing ghost fields, which makes spin-2 algebra anomaly free in such a way that a complete action becomes invariant under non-linear $\hat{W}_{\infty}$ algebra. We call such models the generalized Liouville and Toda conformal models. Extension of Liouville model of this type has been considered in [8] where W-symmetry was discussed.

The main results of this paper are to be found in section 4, where we provide Lagrangians of the generalized Liouville and Toda conformal models. There we establish the precise form of $\hat{W}_{\infty}$ field transformations under which these models are invariant.

\section{Second Bracket Structure of KP Hierarchy}

We will study in this section the second-bracket structure within the general KP setting with the Lax operator:

$$
L \equiv D+\sum_{i=0}^{\infty} W_{i}(x, t) D^{-1-i}
$$

where $D=\partial / \partial x$ acts as an operator on quantities to the right of it. Let us first introduce the second bracket structure as proposed by Gelfand and Dickey (see e.g. [9] for a review). Define $X=\sum_{i=0}^{\infty} D^{i} x_{i}$ and the pairing

$$
\langle L \mid X\rangle \equiv \operatorname{Tr}(L X)=\int \operatorname{res}(L X)=\int \sum_{k=0}^{\infty} W_{k} x_{k}
$$

The Gelfand-Dickey second bracket structure is defined as

$$
\{\langle L \mid X\rangle,\langle L \mid Y\rangle\}_{2}^{G D} \equiv\left\langle X \mid L(Y L)_{-}-(L Y)_{-} L\right\rangle
$$

One obtains from (3) by direct calculation (see for instance [2]):

$$
\left\{W_{n}(x), W_{m}(y)\right\}_{2}^{G D}=\Omega_{n, m}^{(1)}(W(x)) \delta(x-y)+\left.\left\{W_{n}(x), W_{m}(y)\right\}_{2}^{G D}\right|_{\text {nonlinear }}
$$


where the form $\Omega_{n, m}^{(r=1)}(W(x))$ can be read from the general expression:

$$
\Omega_{n, m}^{(r)}(W(x)) \equiv-\sum_{k=0}^{n+r}(-1)^{k}\left(\begin{array}{c}
n+r \\
k
\end{array}\right) W_{n+m+r-k}(x) D_{x}^{k}+\sum_{k=0}^{m+r}\left(\begin{array}{c}
m+r \\
k
\end{array}\right) D_{x}^{k} W_{n+m+r-k}(x)
$$

This type of generalized forms (5) reproduces the linear part of several higher brackets. The nonlinear part in (4) is given by

$$
\begin{aligned}
& \left.\left\{W_{n}(x), W_{m}(y)\right\}_{2}^{G D}\right|_{\text {nonlinear }}=\sum_{i=0}^{m-1}\left[\sum_{k=1}^{m-i-1}\left(\begin{array}{c}
m-i-1 \\
k
\end{array}\right) W_{i}(x) D_{x}^{k} W_{m+n-i-k-1}(x)\right. \\
& \left.-\sum_{k=1}^{n}(-1)^{k}\left(\begin{array}{l}
n \\
k
\end{array}\right) W_{n+i-k}(x) D_{x}^{k} W_{m-i-1}(x)\right] \delta(x-y) \\
& \quad-\sum_{i=0}^{m-1} \sum_{k=0}^{n} \sum_{l=1}^{m-i-1}(-1)^{k}\left(\begin{array}{c}
n \\
k
\end{array}\right)\left(\begin{array}{c}
m-i-1 \\
l
\end{array}\right) W_{n+i-k}(x) D_{x}^{k+l} W_{m-i-l-1}(x) \delta(x-y)
\end{aligned}
$$

So far we had only defined Gelfand-Dickey bracket structures. In order to reproduce the Hamiltonian structure corresponding to KP hierarchy flow equation one realizes that a further structure is required. This structure can be obtained through the Dirac procedure and is called a Drinfeld-Sokolov (DS) bracket. It is given by

$$
\left\{W_{n}(x), W_{m}(y)\right\}^{D S}=-\sum_{i=0}^{n-1} \sum_{j=0}^{m-1}(-1)^{n-i}\left(\begin{array}{c}
n \\
i
\end{array}\right)\left(\begin{array}{c}
m \\
j
\end{array}\right) W_{i}(x) D_{x}^{n+m-i-j-1} W_{j}(x) \delta(x-y)
$$

In conclusion the second Hamiltonian structure compatible with Lenard relations is given by [2]:

$$
\left\{W_{n}(x), W_{m}(y)\right\}_{2}=\left\{W_{n}(x), W_{m}(y)\right\}_{2}^{G D}+\left\{W_{n}(x), W_{m}(y)\right\}^{D S}
$$

We will now discuss realization of the second KP bracket structure in terms of two related two-boson KP systems.

\section{Two-Boson KP Systems and their Miura Relations}

Faá di Bruno Hierarchy. Consider truncated elements of KP hierarchy of the type $L_{J}=$ $D-J+\bar{J} D^{-1}$, where we have introduced two Bose currents $(\bar{J}, J)$ [5]. It has been shown that such two-boson $(\bar{J}, J)$ model is a consistent restriction of the full KP hierarchy [10, 11]. A calculation of the Poisson bracket yields the first bracket structure of two-boson $(\bar{J}, J)$ system given by $\{J(x), \bar{J}(y)\}_{1}=-\delta^{\prime}(x-y)$ and zero otherwise.

One easily associates the two-boson KP hierarchy with so called Faá di Bruno polynomials. The trick is to introduce the gauge transformation generated by $\Phi$ such that $\Phi^{\prime}=J$ :

$$
L_{J}^{\prime}=e^{-\Phi} L_{J} e^{\Phi}=D+\bar{J}(D+J)^{-1}=D+\sum_{n=0}^{\infty}(-1)^{n} \bar{J} P_{n}(J) D^{-1-n}
$$

where $P_{n}(J)=(D+J)^{n} \cdot 1$ are the Faá di Bruno polynomials. As proven in [11] such gauge transformations as in (9) are symplectic and therefore we conclude that

$$
W_{n}=(-1)^{n} \bar{J} P_{n}(J)
$$


satisfy for the first bracket the Poisson-bracket structure of the linear $\mathbf{W}_{1+\infty}$ algebra type, namely $\left\{W_{n}(x), W_{m}(x)\right\}_{1}=\Omega_{n m}^{(0)}(W(x)) \delta(x-y)$ described by the form $\Omega^{(0)}$ given in (5) in its general form.

It is possible to introduce a deformation parameter into the Faá di Bruno representation of $W_{1+\infty}$ algebra by redefining $W_{n}$ to $W_{n}(h)=(-1)^{n} \bar{J}(h D+J)^{n} \cdot 1$ and accordingly $\Omega^{(r)}$ [5]. The semiclassical limit is simply obtained by taking $h \rightarrow 0$ in $W_{n}(h)$ and yields the generators of area preserving $\mathbf{w}_{\mathbf{1}+\infty}$ algebra.

The higher bracket structures have been investigated in [10, 5]. We write the second bracket in the form:

$$
\begin{aligned}
& \{\bar{J}(x), J(y)\}_{2}=J(x) \delta^{\prime}(x-y)-h \delta^{\prime \prime}(x-y) \\
& \{\bar{J}(x), \bar{J}(y)\}_{2}=2 \bar{J}(x) \delta^{\prime}(x-y)+\bar{J}^{\prime}(x) \delta(x-y) \\
& \{J(x), J(y)\}_{2}=c \delta^{\prime}(x-y)
\end{aligned}
$$

Although Lenard relations require that the constants $h$ and $c$ are fixed to $h=1$ and $c=2$ we keep them general in (11) in order to be able to interpret them as independent deformation parameters. This will simplify our discussion in what follows. Among the Poisson bracket structures only first and second are independent, the higher bracket can be given in terms of the first two by means of the recurrence relations [5].

Second Bracket from Two-Boson KP System. We will now show how to generate the second bracket structure from the representation given by (10). The algebra of $J$ and $\bar{J}$ will be given by (11). Here we take $h=1$. Letting again $\Phi$ be such that $\Phi^{\prime}(x)=J(x)$ we derive from (11):

$$
\{\bar{J}(x), \exp ( \pm \Phi(y))\}_{2}=\mp \delta(x-y) \partial \exp ( \pm \Phi(y)) \pm \delta^{\prime}(x-y) \exp ( \pm \Phi(y))
$$

repeating similar calculation as in [5] we get for the linear part of $\{\cdot, \cdot\}_{2}$ the expected result $\left.\left\{W_{n}(x), W_{m}(y)\right\}_{2}\right|_{\text {linear }}=\Omega_{n m}^{(1)}(W(x)) \delta(x-y)$. To calculate the nonlinear part of the bracket we will use the exponential representation of Faá di Bruno polynomials $P_{n}(J)=$ $\exp (-\Phi) \partial^{n} \exp (\Phi)$. We first observe that

$$
\{\Phi(x), \Phi(y)\}_{2}=-c \varepsilon(x-y)
$$

from which, the direct calculation yields

$$
\begin{aligned}
& \left\{P_{n}(x), P_{m}(y)\right\}_{2}=-c\left[\sum_{l=0}^{n} \sum_{p=0}^{m}\left(\begin{array}{c}
n \\
l
\end{array}\right)\left(\begin{array}{c}
m \\
p
\end{array}\right) P_{n-l}(x) P_{m-p}(y) \partial_{x}^{l} \partial_{y}^{p}\right. \\
& \quad-P_{n}(x) \sum_{l=0}^{m}\left(\begin{array}{c}
m \\
l
\end{array}\right) P_{m-l}(y) \partial_{y}^{l}-P_{m}(y) \sum_{l=0}^{n}\left(\begin{array}{c}
n \\
l
\end{array}\right) P_{n-l}(x) \partial_{x}^{l} \\
& \left.\quad+\quad P_{n}(x) P_{m}(y)\right] \varepsilon(x-y)
\end{aligned}
$$

where we wrote for brevity $P_{n}(J(x))=P_{n}(x)$. In the final expression pure $\varepsilon(x-y)$ terms cancel out leaving only delta functions and their derivatives:

$$
\left\{P_{n}(x), P_{n}(y)\right\}_{2}=-c\left[\sum_{l=1}^{n} \sum_{p=1}^{m}(-1)^{p}\left(\begin{array}{c}
n \\
l
\end{array}\right)\left(\begin{array}{c}
m \\
p
\end{array}\right) P_{n-l}(x) P_{m-p}(y) \partial_{x}^{l+p-1}\right] \delta(x-y)
$$


We obtain therefore the complete second bracket for the generators in (10) as the sum of linear and nonlinear terms (after a change of variables $n-l=i, m-p=j$ in (15)):

$$
\begin{aligned}
& \left\{W_{n}(x), W_{m}(y)\right\}_{2}=\Omega_{n m}^{(1)}(W(x)) \delta(x-y) \\
& -c\left[\sum_{i=0}^{n-1} \sum_{j=0}^{m-1}(-1)^{n-i}\left(\begin{array}{c}
n \\
i
\end{array}\right)\left(\begin{array}{c}
m \\
j
\end{array}\right) W_{i}(x) D_{x}^{n+m-i-j-1} W_{j}(x)\right] \delta(x-y)
\end{aligned}
$$

for $n, m \geq 0$.

One recognizes in the second term on the right hand side of (16) only the DS structure from (7) multiplied by $c$ while the nonlinear part of the second Gelfand-Dickey bracket from (田) appears to be missing. This rises the question whether we have an agreement with the second Hamiltonian structure of (8) for our basis (10). The following identity

$$
\left.\left\{W_{n}(x), W_{m}(y)\right\}_{2}^{G D}\right|_{\text {nonlinear }}=\left\{W_{n}(x), W_{m}(y)\right\}^{D S}
$$

valid for the generators $W_{n}(x)=(-1)^{n} \bar{J}(x) P_{n}(J(x))$ shows that this is indeed the case (for the proof see [5]).

As a consequence we are able to rewrite relation (16) for the special case of $c=2$ as

$$
\left\{W_{n}(x), W_{m}(y)\right\}_{2}=\left\{W_{n}(x), W_{m}(y)\right\}_{2}^{G D}+\left\{W_{n}(x), W_{m}(y)\right\}^{D S}
$$

where on the right hand side of (18) we had split nonlinear part of (16) equally between $\left.\{\cdot, \cdot\}_{2}^{G D}\right|_{\text {nonlinear }}$ and $\{\cdot, \cdot\}^{D S}$.

Quadratic Two-Boson KP Hierarchy. Here we call quadratic two-boson KP hierarchy the model based on the pseudo-differential operator [4]:

$$
L_{\jmath}=D+\bar{\jmath}(D-\jmath-\bar{\jmath})^{-1} \jmath
$$

Let us discuss the Poisson bracket structure first. Among the Poisson bracket structures only second and third are local. The non-vanishing bracket within the second structure is given by

$$
\{\jmath(x), \bar{\jmath}(y)\}_{2}=\delta^{\prime}(x-y)
$$

As shown in [12] the Hamiltonian structure corresponding to the Lax operator $L_{\jmath}$ in (19) is invariant under the transformation $g$ and its inverse $g^{-1}$

$$
\begin{aligned}
g(\jmath)=\bar{\jmath}-\frac{\jmath^{\prime}}{\jmath} & , & g(\bar{\jmath})=\jmath \\
g^{-1}(\bar{\jmath})=\jmath+\frac{\bar{\jmath}^{\prime}}{\bar{\jmath}} & , & g^{-1}(\jmath)=\bar{\jmath}
\end{aligned}
$$

Hence we conclude that the Lax operator given by e.g.

$$
L_{\jmath}=D+\left(\jmath+\frac{\bar{\jmath}^{\prime}}{\bar{\jmath}}\right)\left(D-\jmath-\bar{\jmath}-\frac{\bar{\jmath}^{\prime}}{\bar{\jmath}}\right)^{-1} \bar{\jmath}
$$


leads to the same Hamiltonian functions as (19).

Gauge Equivalence between Faá di Bruno and Quadratic Two-Boson Hierarchies and Generalized Miura Maps. We apply on $L_{\jmath}$ from (19) the gauge transformation generated by $\chi=(\phi+\bar{\phi}-\ln \jmath)$ with result:

$$
L_{\jmath} \rightarrow \exp (-\chi) L_{\jmath} \exp (\chi)=D+\jmath+\bar{\jmath}+\jmath\left(\jmath^{-1}\right)^{\prime}+\bar{\jmath} \jmath D^{-1}=D-J+\bar{J} D^{-1}
$$

where we have introduced

$$
J=-\jmath-\bar{\jmath}+\frac{\jmath^{\prime}}{\jmath} \quad ; \quad \bar{J}=\bar{\jmath} \jmath
$$

One can now verify explicitly that with the bracket structure given by (20) the variables defined in (25) satisfy the second bracket structure (11) of Faá di Bruno hierarchy. Hence we obtained a Miura transform for two-Bose hierarchies in form of (25), which generalizes the usual Miura transformation between one-bose KdV and mKdV structures [12]. As a corollary we also obtained in this way a short proof for the quadratic two-boson KP hierarchy system realizing $\hat{W}_{\infty}$.

With the Lax operator from (23) we find $L_{\jmath}=\bar{\jmath}^{-1} \bar{\jmath} L_{\jmath} \bar{\jmath}^{-1} \bar{\jmath}=D+\left(\bar{\jmath} \jmath+\bar{\jmath}^{\prime}\right)(D-\jmath-\bar{\jmath})^{-1}$. The appropriate gauge transformation gives

$$
L_{\jmath} \sim \exp (-\phi-\bar{\phi}) L_{\jmath} \exp (\phi+\bar{\phi})=D+\jmath+\bar{\jmath}+\left(\bar{\jmath} \jmath+\bar{\jmath}^{\prime}\right) D^{-1}
$$

producing object in Faá di Bruno hierarchy with $J=-\jmath-\bar{\jmath}$ and $\bar{J}=\bar{\jmath} \jmath+\bar{\jmath}^{\prime}$. Note, that under $g^{-1}$ from (22) these variables are transformed into $J=-\jmath-\bar{\jmath}+\frac{\jmath \prime}{\jmath}$ and $\bar{J}=\bar{\jmath} \jmath$ identical to (25).

We see that because of (21) and (22) there is an ambiguity in the possible form of generalized Miura transformation and (25) can also appear in other forms. All of them are connecting the Poisson bracket structure of Faá di Bruno hierarchy with the corresponding Poisson bracket structure of the quadratic two-boson hierarchy.

\section{Lagrangian Models and their W-Infinity Invariance}

Given is a generalized Liouville Lagrangian:

$$
\mathcal{L}=\partial_{+} \phi_{-} \partial_{-} \phi_{-}-\partial_{+} \phi_{+} \partial_{-} \phi_{+}+\kappa e^{2 \phi_{-}}
$$

which differs from the one considered in [8] by sign in front of the second term. We shall take $x_{-}$to be "time" and $x_{+}$to be "space" variables. In this setting contribution to the Hamiltonian density comes entirely from the last term (exponential interaction term). Therefore the classical Hamiltonian density is bounded no matter what signs are in front of the kinetic terms. Lagrangian (27) leads to the following canonical (Dirac) bracket structure:

$$
\left\{\phi_{ \pm}(x), \phi_{ \pm}(y)\right\}=\mp \frac{1}{4} \varepsilon(x-y)
$$


which reproduces the second bracket (20) of the quadratic two-boson hierarchy when we set:

$$
\jmath=\partial_{+}\left(\phi_{+}-\phi_{-}\right) \quad ; \quad \bar{\jmath}=\partial_{+}\left(\phi_{+}+\phi_{-}\right)
$$

Furthermore, in this representation we can find via the generalized Miura transformation given below eq. (26) the elements $\bar{J}, J$ of the Faá di Bruno hierarchy as

$$
\begin{aligned}
& \bar{J}=\bar{\jmath} \jmath+\bar{\jmath}^{\prime}=\left[\left(\partial_{+} \phi_{+}\right)^{2}-\left(\partial_{-} \phi_{-}\right)^{2}\right]+\left(\partial_{+}^{2} \phi_{+}+\partial_{+}^{2} \phi_{-}\right) \\
& J=-\jmath-\bar{\jmath}=-2 \partial_{+} \phi_{+}
\end{aligned}
$$

Recall now that $\bar{J}$ and $J$ are building blocks of the generators $W_{n}=(-1)^{n} \bar{J} P_{n}(J)$ of $\hat{\mathrm{W}}_{\infty}$ algebra (8).

Proposition. The generalized Liouville Lagrangian (27) is invariant under $\hat{W}_{\infty}$ symmetry transformations:

$$
\begin{aligned}
\delta^{(n)} \phi_{+} & =\left\{\int \epsilon W_{n}, \phi_{+}\right\}=\sum_{k=0}^{n-1}\left(\begin{array}{c}
n \\
k+1
\end{array}\right)(-1)^{k+n} \partial_{+}^{k}\left(\epsilon \bar{J} P_{n-k-1}(J)\right) \\
+ & (-1)^{n} \frac{1}{2}\left[\epsilon P_{n}(J) J+\partial_{+}\left(\epsilon P_{n}(J)\right)\right] \quad n=0,1,2, \ldots \\
\delta^{(n)} \phi_{-} & =\left\{\int \epsilon W_{n}, \phi_{-}\right\}=(-1)^{n+1}\left[\epsilon P_{n}(J) \partial_{+} \phi_{-}+\frac{1}{2} \partial_{+}\left(\epsilon P_{n}(J)\right)\right]
\end{aligned}
$$

with $\partial_{-} \epsilon=0$.

Proof. First note that the interaction term $\int V=\int \kappa e^{\phi_{-}}$is invariant independently of the remaining kinetic terms in $(27)$ as follows from $(33)$ :

$$
\delta^{(n)} V=2 \kappa\left(\delta^{(n)} \phi_{-}\right) e^{2 \phi_{-}}=(-1)^{n+1} \kappa \partial_{+}\left[\epsilon P_{n}(J) e^{2 \phi_{-}}\right]
$$

The rest of the proof follows by inspection. It is in fact easy to check that combination of two kinetic terms (but not single terms separately) in (27) is invariant. This requires only one technical identity:

$$
\partial_{-} P_{n}(J)=-2 \sum_{k=0}^{n-1}\left(\begin{array}{c}
n \\
k+1
\end{array}\right)\left(\partial_{+}^{k+1} \partial_{-} \phi_{+}\right) P_{n-k-1}(J)
$$

where we used that from (31) we can write the Faá di Bruno polynomials as $P_{n}(J)=$ $\exp \left(\phi_{+}\right) \partial_{+}^{n} \exp \left(-\phi_{+}\right)$.

Since the symmetry transformations in (32) and (33) are generated by $W_{n}$ 's from (10) this establishes invariance of the generalized Liouville action (27) under $\hat{W}_{\infty}$ algebra.

As an example of (33) and (32) let us take the case of $n=0$ for which we find

$$
\begin{aligned}
& \delta^{(0)} \phi_{+}=-\epsilon \partial_{+} \phi_{+}+\frac{1}{2} \partial_{+} \epsilon \\
& \delta^{(0)} \phi_{-}=-\epsilon \partial_{+} \phi_{-}-\frac{1}{2} \partial_{+} \epsilon
\end{aligned}
$$

We note, that $\exp \left(2 \phi_{ \pm}\right)$transform according to (36) and (37) as conformal primary fields of weights $\mp 1$, respectively. We also note, that $\delta^{(n)}$ transformation of $\phi_{-}$can be viewed as a conformal transformation with the field dependent parameter, namely $\epsilon \rightarrow \epsilon P_{n}(J)$. 
We now generalize the previous construction to the setting of Toda theory associated to a finite Lie algebra $\mathcal{G}$ :

$$
\mathcal{L}=\frac{1}{2} \sum_{a, b}^{r} \eta_{a b}\left(\partial_{+} \phi_{-}^{a} \partial_{-} \phi_{-}^{b}-\partial_{+} \phi_{+}^{a} \partial_{-} \phi_{+}^{b}\right)+\kappa \sum_{a}^{r} \frac{2}{\alpha_{a}^{2}} e^{K_{a b} \phi_{-}^{b}}
$$

where $K_{a b}=2 \alpha_{a} \cdot \alpha_{b} / \alpha_{b}^{2}$ is the Cartan matrix of $\mathcal{G}$, furthermore $\eta^{a b}=\frac{\alpha_{b}^{2}}{2}\left(K^{-1}\right)^{a b}$, while $\alpha_{a}$ denote the simple roots of $\mathcal{G}$. The corresponding brackets are

$$
\left\{\phi_{ \pm}^{a}(x), \phi_{ \pm}^{b}(y)\right\}=\mp \frac{1}{2} \varepsilon(x-y) \eta^{a b}
$$

Next, we construct two-currents $\bar{J}, J$ :

$$
\begin{aligned}
\bar{J} & =\frac{1}{2} \sum_{a, b}^{r} \frac{2}{\alpha_{a}^{2}} K_{a b}\left[\partial_{+} \phi_{+}^{a} \partial_{+} \phi_{+}^{b}-\partial_{+} \phi_{-}^{a} \partial_{+} \phi_{-}^{b}\right]+\sum_{a}^{r} \frac{2}{\alpha_{a}^{2}}\left[\partial_{+}^{2} \phi_{+}^{a}+\partial_{+}^{2} \phi_{-}^{a}\right] \\
J & =\sum_{a}^{r} \Gamma_{a} \partial_{+} \phi_{+}^{a}
\end{aligned}
$$

where we introduced constants $\Gamma_{a}$ to be determined later. Construction of $\bar{J}$ is reminiscent of that of E-M tensor and signs are chosen in such a way that the corresponding Virasoro algebra is centerless. The currents $\bar{J}, J$ enter the algebra (11) with constants

$$
h=-\sum_{a, b}^{r} \frac{2}{\alpha_{a}^{2}} \Gamma_{b} \eta^{a b}=-\sum_{a}^{r} \Gamma_{a} r^{a} \quad ; \quad c=\sum_{a, b}^{r} \Gamma_{a} \Gamma_{b} \eta^{a b}
$$

where $r^{a}=\sum_{b}\left(K^{-1}\right)_{a b}$ are the conformal weights of $\exp \left(-\phi_{-}^{a}\right)$ 13. We can choose $\Gamma_{a}=$ $-K_{m a}$, for fixed but arbitrary $m$, corresponding to a long simple root $\alpha_{m}$ with $\alpha_{m}^{2}=2$ of the corresponding Lie algebra $\mathcal{G}$. In this case, recalling that $\eta^{a b}=\left(\alpha_{b}^{2} / 2\right)\left(K^{-1}\right)_{a b}$, it follows that $h=1$ (with $h$ coinciding with conformal weights of vertices $\exp \left(K_{a b} \phi_{-}^{b}\right)$ ). Furthermore, it also follows that $c=K_{m m}\left(\alpha_{m}^{2} / 2\right)=2$. Hence for an arbitrary Lie algebra $\mathcal{G}$, currents $\bar{J}, J$ will satisfy (11) with $h=1$ and $c=2$ leading to the $\hat{W}_{\infty}$ algebra of the form (18).

For symmetry transformations we find in this case:

$$
\begin{aligned}
\delta^{(n)} \phi_{+}^{c} & =\left\{\int \epsilon W_{n}, \phi_{+}^{c}\right\}=\sum_{k=0}^{n-1}(-1)^{k+n+1}\left(\begin{array}{c}
n \\
k+1
\end{array}\right) \sum_{b}^{r} \Gamma_{b} \eta^{b c} \partial_{+}^{k}\left(\epsilon \bar{J} P_{n-k-1}(J)\right) \\
& +(-1)^{n+1} \epsilon P_{n}(J) \partial_{+} \phi_{+}^{c}+(-1)^{n} \sum_{a}^{r} \frac{2}{\alpha_{a}^{2}} \eta^{a c} \partial_{+}\left(\epsilon P_{n}(J)\right) \\
\delta^{(n)} \phi_{-}^{c} & =\left\{\int \epsilon W_{n}, \phi_{-}\right\}=(-1)^{n+1}\left(\epsilon P_{n}(J) \partial_{+} \phi_{-}^{c}+\sum_{a}^{r} \frac{2}{\alpha_{a}^{2}} \eta^{a c} \partial_{+}\left(\epsilon P_{n}(J)\right)\right)
\end{aligned}
$$

where generators $W_{n}$ have the same functional form as in (10) in terms of currents $\bar{J}, J$ from (40) and (41). As before the vertex part is invariant due to:

$$
\delta^{(n)}\left[\sum_{a}^{r} \frac{2}{\alpha_{a}^{2}} e^{K_{a b} \phi_{-}^{b}}\right]=(-1)^{n+1} \sum_{a}^{r} \frac{2}{\alpha_{a}^{2}}\left[\epsilon P_{n}(J)\left(\partial_{+} e^{K_{a b} \phi_{-}^{b}}\right)+\left(\partial_{+} \epsilon P_{n}(J)\right) e^{K_{a b} \phi_{-}^{b}}\right]
$$


being obviously a total derivative. Proof that the remaining free part of Langrangian is invariant is a simple generalization of the proof given above for invariance of the free part of the generalized Liouville action with the technical identity (35) being now replaced by

$$
\partial_{-} P_{n}(J)=\sum_{a}^{r} \Gamma_{a} \sum_{k=0}^{n-1}\left(\begin{array}{c}
n \\
k+1
\end{array}\right)\left(\partial_{+}^{k+1} \partial_{-} \phi_{+}^{a}\right) P_{n-k-1}(J)
$$

\section{Discussion}

Several remarks are in order.

Since the symmetry is non-linear the knowledge of exact functional form of the generators is required to determine all transformations $\delta^{(n)} \phi_{ \pm}$. This knowledge seems necessary even if one tries to find higher transformations of $\phi_{ \pm}$just by commuting the lower ones (the argument usually used in the case of linear symmetry) due to non-linearity.

With the minus sign in front of the second term in (27) the field $\phi_{+}$appears to be a ghost field introduced to enhance the symmetry structure of the original (conformal) Liouville model. Any attempt to change this minus sign to a plus sign will either render the algebra of $\bar{J}$ anomalous (breaking the above symmetry structure) or will result in introduction of the imaginary constants in the definition of the symmetry transformations.

The models defined by (27) and (38) are integrable. Recall namely the definition of infinitely many charges in involutions $H_{n}(\bar{J}, J)$ associated with two-boson Faá di Bruno hierarchy (see e.g. [12]). They all commute with the vertex Hamiltonians of (27) and (38) and are therefore conserved. This result follows from the bracket relations:

$$
\{J(x), V(y)\}=0 \quad ; \quad\{\bar{J}(x), V(y)\}=V(x) \delta^{\prime}(x-y)
$$

which hold both for the vertex $V(y)=e^{2 \phi_{-}(y)}$ of the generalized Liouville model as well as for the vertices $V(y)=\kappa \sum_{a}^{r} \frac{2}{\alpha_{a}^{2}} e^{K_{a b} \phi_{-}^{b}(y)}$ of the generalized Toda model. Hence both models are integrable and the underlying structure of the two-boson KP hierarchy allows for explicit construction of charges.

We have seen that the symmetry transformations: $g(\jmath)=\bar{\jmath}-(\ln \jmath)^{\prime}, g(\bar{\jmath})=\jmath$ as well as its inverse $g^{-1}$ keep all Hamiltonians invariant. By acting with $g$ and $g^{-1}$ and their powers one obtains the family of vertices: $\jmath \exp \left(-2 \phi_{-}\right), \bar{\jmath} \exp \left(-2 \phi_{-}\right), \ldots$ etc. They will all commute with charges $H_{n}$. Moreover it is possible to associate to each of these vertices a Lagrangian density invariant under $\hat{W}_{\infty}$ but with kinetic terms containing $\phi_{ \pm}$transformed by the corresponding $g$ transformations. One obtains in this process instead of the single Lagrangian (27) a string of Liouville like actions, which reflect existence of the symmetry $g$ in the underlying two-boson KP hierarchy. For instance acting once with $g$ on Lagrangian in (27) leads to:

$$
\mathcal{L}=\partial_{+} \phi_{-} \partial_{-} \phi_{-}-\partial_{+} \phi_{+} \partial_{-} \phi_{+}+\frac{\partial_{+}\left[\partial_{+}\left(\phi_{+}-\phi_{-}\right) \partial_{-}\left(\phi_{+}-\phi_{-}\right)\right]}{2 \partial_{+}\left(\phi_{+}-\phi_{-}\right)}+\kappa \partial_{+}\left(\phi_{+}-\phi_{-}\right) e^{-2 \phi_{-}}
$$

which also is $\hat{\mathrm{W}}_{\infty}$ invariant with $\bar{J}$ and $J$ realized as in (25). 
As it was shown in [12] taking the Dirac constraint $J=0$ reduces the two-boson hierarchies to KdV and mKdV systems. Correspondingly removing $\phi_{+}$in (27) casts the Lagrangian to the form of the usual Liouville model. While the vertex is still commuting with Hamiltonians of the reduced systems $(\mathrm{KdV})$ the corresponding symmetry structure shrinks to the conformal symmetry generated by the reduced current $\bar{J}=-\jmath^{2}+\jmath^{\prime}$.

Acknowledgements We gratefully acknowledge support within CNPq/NSF Cooperative Science Program. One of us (HA) thanks Instituto de Física Teórica-UNESP for kind hospitality.

\section{References}

[1] K. Yamagishi, Phys. Lett. 254B (1991) 436; F. Yu and Y.-S. Wu, Phys. Lett. 263B (1991) 220

[2] A. Das, W.J. Huang and S. Panda, Phys. Lett. 271B (1991) 109

[3] F. Yu and Y.-S. Wu, Nucl. Phys. B273 (1992) 713

[4] F. Yu and Y.-S. Wu, Phys. Rev. Lett. 68 (1992) 2996 (also in hep-th/9112009)

[5] H. Aratyn, L.A. Ferreira, J.F. Gomes and A.H. Zimerman, On Two-Current Realization of KP Hierarchy, hep-th/9206096, to appear in Nucl Phys B; see also Phys. Lett. 293B (1992) 67 (also in hep-th/9201024)

[6] O. Babelon and L. Bonora, Phys. Lett. B244, (1990) 220.

[7] H. Aratyn, L.A. Ferreira, J.F. Gomes and A.H. Zimerman, Phys. Lett. B 254, (1991) 372.

[8] S. Bellucci and E. Ivanov, Mod. Phys. Lett. A6 (1991) 1269

[9] L.A. Dickey, Soliton Equations and Hamiltonian Systems, World Scientific, Singapore (1991); R. W. Carroll, Topics in Soliton Theory, North-Holland, Amsterdam (1991)

[10] B.A. Kupershmidt, Commun. Math. Phys. 99 (1985) 51

[11] H. Aratyn, E. Nissimov, S. Pacheva and I. Vaysburd, Phys. Lett. 294B (1992) 167 (also in hep-th/9209006)

[12] H. Aratyn, L.A. Ferreira, J.F. Gomes, R.T. Medeiros and A.H. Zimerman, Generalized Miura Transformations, Two boson KP hierarchies and their reduction to KdV hierarchies, IFT preprint P-011/93, hep-th/9302125

[13] C.P. Constantinidis, L.A. Ferreira, J.F. Gomes and A.H. Zimerman, Phys. Lett. 298B (1993) 88 (also in hep-th/9207061) 